\title{
Biotechnologies végétales et gestion durable des résistances face à des stress biotiques et abiotiques : avancées et enjeux
}

\author{
Serge Hamon ${ }^{1}$ \\ Maria Manzanares-Dauleux ${ }^{2}$ \\ Didier Picard ${ }^{3}$ \\ ${ }^{1}$ IRD \\ UMR DIAPC, \\ 911, avenue. Agropolis, \\ BP 64501 \\ F-34394 Montpellier \\ France \\ <serge.hamon@ird.fr> \\ 2 UMR 118 APBV \\ Agrocampus Ouest \\ F-35042 Rennes \\ France \\ <Maria.Manzanares@agrocampus-ouest.fr> \\ <maria.manzanares@rennes.inra.fr> \\ ${ }^{3}$ Inra \\ UMR 211 Agronomie \\ BP 01 \\ F-78850 Grignon \\ France \\ <Didier.Picard@grignon.inra.fr>
}

\section{L}

es biotechnologies, au sens large, sont à juste titre mises en avant d'innovations majeures pour l'agriculture de demain. En juillet 2008, se réunissait à Rennes le réseau $\mathrm{AUF}^{1}$ "Biotechnologies Végétales, amélioration des plantes et sécurité alimentaire - BIOVEG", afin de faire un point sur le thème: "Gestion durable des résistances face à des stress biotiques et abiotiques " (encadré). Les textes qui suivent reprennent quelques-unes des conférences prononcées à cette occasion. Plusieurs enseignements généraux peuvent être tirés des exposés et des discussions qui ont suivi à l'occasion de ce colloque.

En premier lieu, la rapidité des progrès effectués dans les connaissances en moins de quarante ans a été soulignée par tous les orateurs, cela dans des domaines aussi divers que la génomique $\left(\right.$ Delseny ${ }^{2}$ ), la caractérisation et la conservation des ressources phytogénétiques (Amirouche, Engelmann), les relations hôte-pathogène ou parasite (Andrivon, Nicole), ou la fixation biologique de l'azote (Hocher) - un des services écologiques très attendus pour améliorer la durabilité des systèmes de production en agriculture. Ces progrès continuent et vont aller en s'accélérant, avec, notamment, le développement des nouvelles techniques de séquençage.

Apparait en même temps la complexité initialement insoupçonnée par les pionniers des biotechnologies et de la génétique - de l'organisation du vivant dans le règne végétal, qui s'est trouvée également illustrée dans l'ensemble des interventions. Par exemple, ce sont les duplications complètes du génome des ancêtres des végétaux actuels qui compliquent singulièrement l'annotation des génomes

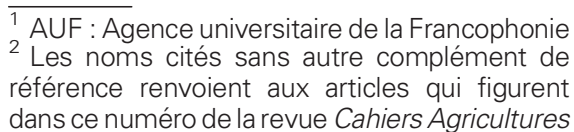

séquencés. Ce sont aussi les gènes déterminant les micro-ARN, découverts récemment et dont le rôle n'est pas encore bien connu (Delseny). Ce sont les mécanismes mis en place dans la reconnaissance hôte micro-organisme, pathogène ou non, ceux impliqués dans les réactions des plantes, d'une diversité prodigieuse (Andrivon). De ce fait, Arabidopsis thaliana n'est plus considéré comme le modèle idéal de génome des dicotylédones - même si cette espèce demeure un outil incontournable par la facilité de sa culture et la diversité de ses écotypes. On s'achemine vers le choix d'une plante modèle par grand groupe phylogénétique. De cette complexité, résulte la diversité des espèces et écotypes présents dans une région (Amirouche), dont une partie pourrait être perdue du fait de l'activité humaine, en raison des faibles moyens mis pour explorer cette diversité.

Cette complexité contribue à expliquer pourquoi des progrès qui avaient été imaginés comme possibles au début de l'essor des biotechnologies n'ont pas encore été obtenus. Une illustration en est donnée par la fixation biologique de l'azote (Hocher) effectuée par un petit nombre d'espèces d'un nombre limité de familles. Cette fonction physiologique, résultat de mécanismes très complexes, liés aux effets de multiples gènes, est extrêmement difficile à maitriser.

Malgré ces difficultés, nul ne doute que les biotechnologies soient désormais incontournables pour progresser. Dans le domaine de l'agriculture et de l'alimentation, les progrès attendus portent sur une agriculture plus durable, tirant un meilleur parti des services écosystémiques évoqués par le Millenium Assessment $^{3}$, et une alimentation plus

\footnotetext{
${ }^{3}$ Millenium Assessment. Voir : www.millenniumassessment.org
} 
équilibrée et plus sûre. Marion Guillou (2007) ${ }^{4}$ écrivait notamment dans l'éditorial qu'elle a signé pour Cabiers Agricultures : "Elles constituent aujourd'bui un outil, aussi indispensable que commun, des laboratoires de biologie et génétique végétale pour leur activité de recherches sur le fonctionnement des plantes et des interactions plante-environnement."
Les innovations issues des travaux dans ce domaine devront cependant être resituées dans le contexte de systèmes de production qui soient adaptés régionalement aux besoins des producteurs et consommateurs et insérés dans les agro-écosystèmes ${ }^{5}$ locaux. Le développement des biotechnologies orientées vers le progrès de l'agriculture devra donc s'inscrire dans le développement plus global des sciences agronomiques, au sens large, pour être apte à faire face aux enjeux qui sont ceux de l'agriculture de demain. Le travail en réseau, au Nord comme au Sud, sur des projets ambitieux, pluridisciplinaires, constitue indéniablement une nécessité pour un véritable avènement des biotechnologies végétales.

\section{Les $\mathrm{XI}^{\mathrm{e}}$ journées scientifiques du réseau de chercheurs de l'Agence universitaire de la Francophonie (AUF)}

Du 30 juin au 3 juillet 2008, Agrocampus Ouest, avec le soutien de l'université de Rennes 1, I'Institut national de recherche agronomique (Inra) et I'Institut pour la recherche et le développement (IRD), a accueilli les XI journées scientifiques du réseau de chercheurs de l'Agence universitaire de la Francophonie (AUF) "Biotechnologies végétales : amélioration des plantes et sécurité alimentaire" sur le thème "Biotechnologies végétales et gestion durable des résistances face à des stress biotiques et abiotiques ". Cette rencontre, où 57 communications, dont 13 introductives ont été présentées, fut l'occasion de faire le point sur les progrès enregistrés ces dernières années dans le domaine très large des biotechnologies végétales, de la culture in vitro à la génomique, avec en perspective une agriculture et un développement durables.

Six sessions thématiques furent proposées portant respectivement sur :

1) la conservation, l'évaluation et la régénération des ressources génétiques ;

2) la résistance des plantes aux bioagresseurs ;

3) les mécanismes de résistance des plantes aux contraintes abiotiques ;

4) les stratégies et outils de sélection pour l'amélioration de la qualité ;

5) le développement d'outils biotechnologiques ;

6) la valorisation des acquis par la création de variétés adaptées.

Les articles qui suivent reprennent la conférence introductive du professeur Delseny ainsi que quelques exposés des sessions $1,2,3$ et 5 .

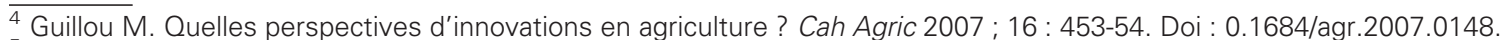

${ }^{5}$ Agro-écosystème pris au sens de l'ensemble constitué d'un ou plusieurs agrosystèmes et d'un ou plusieurs écosystèmes juxtaposés et en interaction les uns avec les autres.
} 